Conclusions: ILD is a serious complication in RA with a significantly increased mortality compared with a large matched cohort of RA comparisons without ILD. Disclosure of Interest: None declared

DOI: 10.1136/annrheumdis-2017-eular.2848

\section{FRI0175 USING SMARTPHONES TO IMPROVE REMOTE MONITORING OF RHEUMATOID ARTHRITIS: COMPLETENESS OF PATIENTS' SYMPTOM REPORTS}

S. Van Der Veer ${ }^{1}$, L. Austin ${ }^{1,2}$, C. Sanders ${ }^{2}$, W. Dixon ${ }^{1} .{ }^{1}$ Arthritis UK Centre for Epidemiology, Centre for Musculoskeletal Research, Faculty of Biology, Medicine and Health, Manchester Academic Health Science Centre, University of Manchester; ${ }^{2}$ Centre for Primary Care, Faculty of Biology, Medicine and Health, Manchester Academic Health Science Centre, University of Manchester, Manchester, United Kingdom

Background: Clinical decisions about management of rheumatoid arthritis (RA) happen at intermittent clinic visits. In the absence of objective measures of disease severity between visits, understanding fluctuating disease severity largely relies on patients' symptom reports, and thus on patients' recall, eloquence, stoicism and willingness to discuss symptoms.

The Remote Monitoring of Rheumatoid Arthritis (REMORA) study aims to improve monitoring of disease severity in RA. Patients, clinicians and researchers codesigned a smartphone app to enable RA patients to report daily symptoms in between clinic visits with data integrated into the electronic health record. The data presented here were collected as part of the REMORA feasibility evaluation. Objectives: To evaluate the completeness of patient-reported symptom data submitted through the REMORA smartphone app over three months.

Methods: We invited 23 RA patients treated at the outpatient clinic of Salford Royal Foundation Trust (UK) to record their symptoms for three months using the REMORA smartphone app. Participants received notifications to record: daily scores for the seven items of the RA Impact of Disease score, as well as morning stiffness on a scale from 0 to 10 ; weekly scores for thirteen items on 28 tender and swollen joint self-assessments, global assessment, impact on work and activity, and flare occurrence; and monthly scores for the Health Assessment Questionnaire (HAQ) 20-item disability scale. We calculated the time that participants were in the study as the number of days between the first and last day of submitting daily scores. We then explored patterns of data entry, as well as entry completeness.

Results: Twenty patients accepted the invitation to participate. Eight (40\%) were male, all but one were white British, and their mean age was $56.9 \pm 11.1$ years. The median number of days in the study was 82 (interquartile range [IQR], 80 to 82). While being in the study, participants submitted daily scores on almost all days (median, $91 \%$ of days; IQR, 78 to 95 ), with four doing this on $<60 \%$ of study days. Across participants, almost all of 1325 daily entries were complete, with only nine $(<1 \%)$ having missing values for up to two individual items. Participants submitted weekly scores for a medium of 11 out of 13 weeks (IQR, 10 to 12). Of all 213 weekly entries, fifteen $(7 \%)$ had missing values, but never more than two. Lastly, 8/20 participants provided monthly HAQ scores only once, while a further $9 / 20$ and $3 / 20$ participants did this for two and all three months, respectively. No monthly entries had any missing values.

Conclusions: Our feasibility study showed that smartphones have the potential to support collection of daily patient reports of symptoms with high levels of completeness over three months. Lengthier monthly question sets were less likely to be completed compared to briefer daily and weekly ones. Future steps include exploring methods for adapting data entry frequency to (fluctuations in) disease severity in order to support sustained symptom reports over longer time periods and in a wider group of patients.

Disclosure of Interest: None declared

DOI: 10.1136/annrheumdis-2017-eular.4243

\section{FRI0176 CORRELATION BETWEEN DISEASE ACTIVITY AND MENTAL HEALTH IN CHINESE PATIENTS WITH RHEUMATOID ARTHRITIS -ASSESSMENT WITH SMART SYSTEM OF DISEASE MANAGEMENT (SSDM) MOBILES TOOLS}

X. Li ${ }^{1}$, H. Sun ${ }^{2}$, R. Wu ${ }^{3}$, H. Wei ${ }^{4}$, W. Fan ${ }^{5}$, C. Zhao ${ }^{6}$, Z. Zhang ${ }^{7}$, Z. Wu ${ }^{8}$, Y. Wang ${ }^{9}$, J. Huang ${ }^{10}$, F. Xiao ${ }^{11}$, H. Xiao ${ }^{11}$, Y. Jia ${ }^{11}$, M. Zhang ${ }^{12} .{ }^{1}$ Dept. of Rheumatology, Anhui Medical University Affiliated Provincial Hospital, Hefei; ${ }^{2}$ Dept. of Rheumatology, Shandong Provincial Hospital, Jinan; ${ }^{3}$ The First Affiliated Hospital of Nanchang University, Nanchang; ${ }^{4}$ Dept. of Rheumatology, Northern Jiangsu People's Hospital, Yangzhou; ${ }^{5}$ Dept. of Rheumatology, Central Hospital of XinXiang, XinXiang; ${ }^{6}$ Dept. of Rheumatology and Immunology, The First Affiliated Hospital of Guangxi Medical University, Nanning: ${ }^{7}$ Linyi people's Hospital, Linyi; ${ }^{8}$ Dept. of Rheumatology and Immunology, The First Affiliated Hospital of The Fourth Military Medical University, Xi'an; ${ }^{9}$ Dept. of Rheumatology and Immunology, The First Affiliated Hospital of BaoTou Medical College, Baotou; ${ }^{10}$ Dept. of Rheumatology and Immunology, The sixth Affiliated Hospital of Sun Yat-sen University, Guangzhou: ${ }^{11}$ Medical Dept., Shanghai Gothic Internet Technology Co., Ltd., Shanghai; ${ }^{12}$ Dept. of Rheumatology and Immunology, Jiangsu Province Hospital, Nanjing, China

Background: Rheumatoid arthritis (RA) patients can cause joint swelling and tenderness, and affect the patient's mental health. Previous studies showed that $13-47 \%$ of RA patients suffered from anxiety and/or depression. Hospital anxiety and depression scale (HADS) is usually used to evaluate patients' mental health, which consists of 14 items divided into an anxiety subscale (HADS-A) and a depression subscale (HADS-D). Disease activity score in 28 joints (DAS28) is one of the most used tool to assess RA patients' disease activity. Both HADS and DAS28 assessments were commonly led by health professionals with paper questionaire. This study applies the mobile platform of Smart System of Disease Management (SSDM) for evaluating DAS28 and HADS by RA patients.

Objectives: The purpose of this study is to describe the morbidity of mental comorbidity in Chinese patients with RA and analyze the potential association among DAS28 and HADS.

Methods: SSDM is a mobile application includes physicians' and patients' interfaces. The patient's terminal system includes self-assessment (DAS28, HADS), lab test results and medication management. After data entry, patients can synchronize data to the mobile terminal of their authorized rheumatologist. A cohort study was conducted with patients who were diagnosed with RA in tertiary hospital across China. All participants self-assessed both DAS28 and HADS with SSDM at least one time. Descriptive statistics were performed for patient and disease characteristics, assuming normality for DAS28 distribution and the level of disease activity was analyzed using Pearson's statistics. One-way analysis of variance was employed to explore for difference between sub-groups. Bivariate correlation and linear logistic analysis were employed to explore for potential correlation between DAS28 and HADS.

Results: From June 2016 to January 2017, 230 patients (male 66, female 164) from 12 hospitals performed 311 times HADS and 517 times DAS28 self-assessment. The mean $( \pm S D)$ age was $34.17 \pm 13.11$ (11-71) years, with the median disease duration of $24.70(0-572)$ months. As the standard of HADS score higher than $10,31 \%$ and $37 \%$ patients could be diagnosed as anxiety and depression respectively. According to the DAS28 assessment results, the proportion of patients with remission, low disease activity, moderate activity and high activity were $18 \%, 19 \%, 46 \%$ and $18 \%$ respectively. Bivariate correlation showed that DAS28 was positively correlated with HADS, $p<0.001$. Both HADS$A$ and HADS-D showed linear regression association with DAS28 score, the regression equation as "HADS-A $=5.962+0.435^{*}$ DAS28" and "HADS-D $=6.379$ $+0.694^{*}$ DAS28" respectively, $\mathrm{p}<0.001$.

Conclusions: DAS28 was positively correlated with HADS. SSDM is an effective mobile interface to serve for RA patients performing self-management of both disease activity and mental health as well as to supply physicians with valuable data.

Disclosure of Interest: None declared

DOI: 10.1136/annrheumdis-2017-eular.4032

\section{FRI0177 THE UNDERRATED PREVALENCE OF DEPRESSION IN PATIENTS WITH RHEUMATOID ARTHRITIS}

R. Sruamsiri ${ }^{1}$, Y. Kaneko ${ }^{2}$, J. Mahlich ${ }^{1} .{ }^{1}$ Health Economics, Janssen Pharmaceutical KK, Tokyo, Japan; ${ }^{2}$ Department of Internal Medicine, Keio University School of Medicine, Tokyo, Japan

Background: Significant evidence in the scholarly literature suggests that depression is a common comorbidity among patients with rheumatoid arthritis (RA) (1) Comorbid depression in RA patients is especially troublesome because it often goes unrecognized and untreated (2) often because rheumatologists and their patients seldom communicate about depression (3). In addition, evidence of depression in RA patients is limited in Asia studies.

Objectives: To determine the prevalence of depression among patients with RA and explore the relationships between depression and an array of variables.

Methods: Cross-sectional online survey $(n=500)$ of RA patients including the Patient Health Questionnaire [PHQ-9] (4) to measure the presence and severity of depressive symptoms were performed. The survey contained patient demographic, clinical characteristics such as functional impairment as assessed using the Japanese version of the Stanford Health Assessment Questionnaire (J-HAQ score), and the participant's current medical treatment. Ordered logistic regression was used to identify the determinants of depression conditions among survey respondents.

Results: The mean age of the 500 patients with RA was 54.3 years old and $67 \%$ were female. While only $25(5 \%)$ of the population studied had been officially diagnosed with depression, 176 (35\%) had PHQ-9 scores indicating depression was present. Comorbidity conditions, except for migraine and heart conditions, were not different between patients with depression and those without. Logistic regression analysis revealed a negative correlation between the prevalence of depression and younger age with odds ratio (OR) of 0.96 (95\% confidence interval (Cl); 0.93-0.98), higher education (OR, $0.60 ; 95 \% \mathrm{Cl}, 0.36-0.98$ for a bachelor's degree or higher) and an income level of $0.8-1.6$ million yen (OR, $0.45 ; 95 \% \mathrm{Cl}$, $0.22-0.91)$. Positive correlations with depression was found in RA patients with high J-HAQ score $(\mathrm{OR}, 1.99 ; 95 \% \mathrm{Cl}, 1.47-2.71)$. Patients treated with biologic monotherapy were significantly less likely to have depression compared to those treated with non-biologic anti-rheumatic drugs (OR, 0.36; 95\% Cl, 0.17-0.75)

Conclusions: It is a potential risk of under-diagnosis and under-reporting of Depression in patients with RARA patients are more likely to experience depression if they are younger, have greater functional impairment, or whose treatment regimen includes pain medications without biologic drugs. 